\title{
A prospective, double-blind, randomized, placebo-controlled study on the efficacy and safety of influenza vaccination in myasthenia gravis
}

\author{
Ellen Strijbos ${ }^{a, *}$, Martijn R. Tannemaat ${ }^{a}$, Iris Alleman ${ }^{b}$, Robert H.P. de Meel ${ }^{a}$, Jaap A. Bakker ${ }^{c}$, \\ Ruud van Beek $^{\mathrm{f}}$, Frank P. Kroon ${ }^{\mathrm{d}}$, Guus F. Rimmelzwaan ${ }^{\mathrm{e}}$, Jan J.G.M. Verschuuren ${ }^{\mathrm{a}}$ \\ a Department of Neurology, Leiden University Medical Center, the Netherlands \\ ${ }^{\mathrm{b}}$ Department of Physiotherapy, Leiden University Medical Center, the Netherlands \\ ${ }^{\mathrm{c}}$ Department of Clinical Chemistry and Laboratory Medicine, Leiden University Medical Center, the Netherlands \\ ${ }^{\mathrm{d}}$ Department of Infectious Diseases, Leiden University Medical Center, the Netherlands \\ ${ }^{\mathrm{e}}$ Research Center for Emerging Infections and Zoonoses, University of Veterinary Medicine, Hannover, Germany \\ ${ }_{\mathrm{f}}^{\mathrm{f}}$ Department of Viroscience, Erasmus Medical Center, Rotterdam, the Netherlands
}

\section{A R T I C L E I N F O}

\section{Article history:}

Received 3 October 2018

Received in revised form 4 January 2019

Accepted 7 January 2019

Available online 16 January 2019

\section{Keywords:}

Influenza

Vaccination

Autoimmune disease

Myasthenia gravis

Immunosuppression

Antibodies

\begin{abstract}
A B S T R A C T
Objective: To investigate the efficacy and safety of an influenza vaccination in patients with myasthenia gravis with acetylcholine receptor antibodies (AChR MG).

Methods: An influenza vaccination or placebo was administered to $47 \mathrm{AChR}$ MG patients. Before and 4 weeks after administration blood samples and clinical outcome scores were obtained. Antibodies to the vaccine strains A/California/7/2009 (H1N1)pdm09, A/Hong Kong/4801/14 (H3N2) and B/ Brisbane/060/08 were measured using the hemagglutination-inhibition (HI) assay and disease-specific AChR antibody titers were measured with a radio-immunoprecipitation assay. Forty-seven healthy controls (HC) were vaccinated with the same influenza vaccine to compare antibody titers.

Results: A post-vaccination, seroprotective titer ( $\mathrm{HI} \geq 1: 40)$ was achieved in $89.4 \%$ of MG patients vs. 93.6\% in healthy controls for the H3N2 strain, 95.7\% vs $97.9 \%$ for the H1N1 strain and 46.8 vs $51 \%$ for the B-strain. A seroprotective titer for all three strains of the seasonal influenza vaccine was reached in $40.4 \%$ (19/47) of the MG group and in 51\% (24/47) of the HC group. Immunosuppressive medication did not significantly influence post geomean titers (GMT). The titers of disease-specific AChR antibodies were unchanged 4 weeks after vaccination. The clinical outcome scores showed no exacerbation of MG symptoms.

Conclusion: The antibody response to an influenza vaccination in patients with AChR MG was not different from that in healthy subjects, even in AChR MG patients using immunosuppressive medication. Influenza vaccination does not induce an immunological or clinical exacerbation of AChR MG.

Clinical trial registry: The influenza trial is listed on clinicaltrialsregister.eu under 2016-003138-26.
\end{abstract}

(c) 2019 Elsevier Ltd. All rights reserved.

\section{Introduction}

Myasthenia gravis (MG) is an acquired autoimmune disease of the neuromuscular junction and is characterized by fluctuating weakness and fatigability of skeletal muscles [1]. In the majority of MG patients acetylcholine receptor (AChR) antibodies are found [1]. Symptomatic treatment is often insufficient, and a considerable proportion of patients need long-term immunosuppressive medication (IM). Patients with an autoimmune disorder are generally believed to be at an increased risk of infection, either due to their immunosuppressive therapy or due to immune abnormalities

\footnotetext{
* Corresponding author at: Albinusdreef 2, 2333 ZA Leiden, the Netherlands. E-mail address: e.strijbos@lumc.nl (E. Strijbos).
}

associated with their disease [2,3]. Conversely, an infection can cause exacerbation of symptoms, potentially resulting in a myasthenic crisis. Specific data on infection rates in myasthenic patients are not available [4].

Little is known about the efficacy and safety of vaccination in patients with autoimmune diseases. No specific guidelines regarding vaccinations in patients with MG exist, but a small number of observational studies suggest that influenza vaccination is safe [5-7] and recently a randomized controlled trial showed that influenza vaccination has no influence on AChR antibody titers [8]. In a recent study we found a small, temporary, but significant increase in Quantitative Myasthenia Gravis scores (QMG) after tetanus vaccination. However, this was far less than what is generally considered clinically relevant [9]. In the Netherlands, annual vaccination 
against influenza is recommended for all patients with an autoimmune disease [10]. However, in our personal experience and as described earlier [5], many patients express concern that vaccination may lead to an exacerbation and a substantial number decline vaccination each year based on these concerns. This is unfortunate, as seasonal vaccination against influenza is highly effective in reducing laboratory-confirmed influenza illness, hospital admissions and risk of death, especially in elderly and frail patients [11]. This is relevant, as this age group has the highest incidence of autoimmune MG [12]. Another concern is that IM may hamper the development of protective antibody levels. Therefore, we performed a double-blind placebo-controlled trial to investigate the efficacy and safety of the seasonal (2016/2017) influenza vaccine in patients with AChR MG with and without IM.

\section{Materials and methods}

\subsection{Standard protocol approvals, registrations, and patient consents}

This study was approved by the Local Committee on Medical Ethics of the LUMC. Subjects provided written informed consent and received reimbursement of travel costs. The trial is listed on clinicaltrialsregister.eu under 2016-003138-26.

\subsection{Patients}

We included 47 patients with AChR MG and 47 healthy controls at the start of the flu season (October 2016). AChR MG patients were recruited from the neurology outpatient clinic of Leiden University Medical Center (LUMC) and through the national patient organization. Seasonal influenza vaccination was offered at the start of the flu season to all LUMC employees; healthy controls were recruited from this population.

Inclusion criteria for the patient group were a diagnosis of AChR MG, age $\geq 18$ years and stable disease in the past 3 months. Diagnosis of AChR MG was based on clinical signs or symptoms consistent with MG and a positive serological test for AChR antibodies. A maximum daily dose of $30 \mathrm{mg}$ of prednisolone, with a variation of $\pm 5 \mathrm{mg}$ during 3 months before participation was allowed as well as use of other immunosuppressive medication. During the study, patients were on a stable dose of their medication (see Table 1). Time from last pyridostigmine dose to clinical testing was kept constant for each patient on test days, but was allowed to vary between patients.

Inclusion criteria for healthy controls were an age $\geq 18$ years and no autoimmune disease or immunosuppressive medication.

Exclusion criteria for the AChR MG group were: instable or severe disease as evidenced by recent changes in medication or an MGFA classification of 4 or 5 , presence of a thymoma, use of vitamin $\mathrm{K}$ antagonist or new oral anti-coagulants (NOACs), pregnancy and other diseases of the immune system that may affect the efficacy of vaccination.

\subsection{Study protocol}

This single-center, prospective, double-blind, randomized, placebo- controlled study was performed at the LUMC. Randomization was performed by a randomization list created by the hospital pharmacy. Patients and physicians performing clinical tests were blinded for treatment allocation until the end of T1. Research nurses, who administered the vaccination, were not blinded, because the placebo was provided in a different syringe than the commercial influenza vaccine. Patients were randomized to receive either an intramuscular injection with the influenza vaccine or a placebo $(0.5 \mathrm{~mL} 0.9 \% \mathrm{NaCl})(\mathrm{T} 0)$. At T0 age, sex, disease duration, use of medication, MGFA classification, thymectomy and seasonal influenza vaccinations in the previous 3 years were recorded. Prior to injection (T0) and four weeks later (T1), serum and several clinical outcome measures were obtained. Four weeks (T1) after this first vaccination, patients were unblinded and patients in the placebo group were vaccinated with the influenza vaccine (Fig. 1). At T2, 4 weeks after the flu vaccination, a third blood sample and MG specific activities of daily living (MG-ADL) score were obtained from the (initial) placebo group. In all patients, an MG-ADL was obtained by phone by a research nurse, twelve weeks after influenza vaccination (T3). At T1, T2 and T3 AChR MG patients were asked for side effects and exacerbation

Table 1

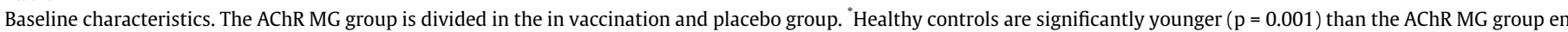
consist out of significantly more females $(\mathrm{p}=0.02)$. ${ }^{* *}$ MGFA classification: Myasthenia gravis foundation America classification.

\begin{tabular}{|c|c|c|c|c|}
\hline & AChR MG Vaccination & AChR MG Placebo & $\mathrm{HC}$ & Total \\
\hline Number of patients $-\mathrm{n}$ & 24 & 23 & 47 & 94 \\
\hline Gender, female (\%) & $11(45.8)$ & $14(60.9)$ & $36(76.6)^{*}$ & $61(64.9)$ \\
\hline Age, median years (range) & $61.5(32-72)$ & $63(22-74)$ & $54(24-65)^{*}$ & \\
\hline Duration of disease, mean years (SD) & $14.3(13.9)$ & $10.7(9.9)$ & - & \\
\hline MGFA classification $^{* *}$ & & & - & \\
\hline $0-\mathrm{n}(\%)$ & $10(41.7)$ & $8(34.8)$ & - & \\
\hline $1-\mathrm{n}(\%)$ & 0 & $5(21.7)$ & - & \\
\hline $2-\mathrm{n}(\%)$ & $13(54.2)$ & $9(39.1)$ & - & \\
\hline $3-n(\%)$ & $1(4.2)$ & $1(4.3)$ & - . & \\
\hline Use of immunosuppressive medication, $\mathrm{n}(\%)$ & $15(62.5)$ & $14(60.9)$ & - & \\
\hline Prednisolone, n (\%) & $9(37.5)$ & $11(47.8)$ & - & \\
\hline Mean daily dose, mg (range) & $9.2(5-20)$ & $6.8(1-10)$ & - & \\
\hline Azathioprine, $\mathrm{n}(\%)$ & $13(54.2)$ & $10(43.5)$ & - & \\
\hline Mean daily dose, mg (range) & $131.2(50-200)$ & $116.7(50-200)$ & - & \\
\hline Mycophenolic acid, $\mathrm{n}(\%)$ & 0 & $2(8.7)$ & - & \\
\hline Mean daily dose, mg (range) & - & $2000(2000)$ & - & \\
\hline Cyclosporine, $\mathrm{n}(\%)$ & $3(12.5)$ & 0 & - & \\
\hline Mean daily dose, $\mathrm{mg}$ (range) & $166.7(150-200)$ & - & - & \\
\hline Combination of immunosuppressive medication, $\mathrm{n}(\%)$ & $8(33.3)$ & $8(34.8)$ & - & \\
\hline Thymectomy in the past ( $>1$ year ago) $-\mathrm{n}(\%)$ & $15(62.5)$ & $14(60.9)$ & - & \\
\hline Past seasonal trivalent inactivated influenza vaccination $-\mathrm{n}(\%)$ & & & $39(83)$ & $78(83)$ \\
\hline $2015-2016$ & $15(62.5)$ & $16(69.6)$ & $28(59.6)$ & $59(63)$ \\
\hline 2014-2015 & $16(66.7)$ & $17(73.9)$ & $33(70.2)$ & $66(70)$ \\
\hline 2013-2014 & $16(66.7)$ & $15(65.2)$ & $31(66)$ & $62(66)$ \\
\hline
\end{tabular}




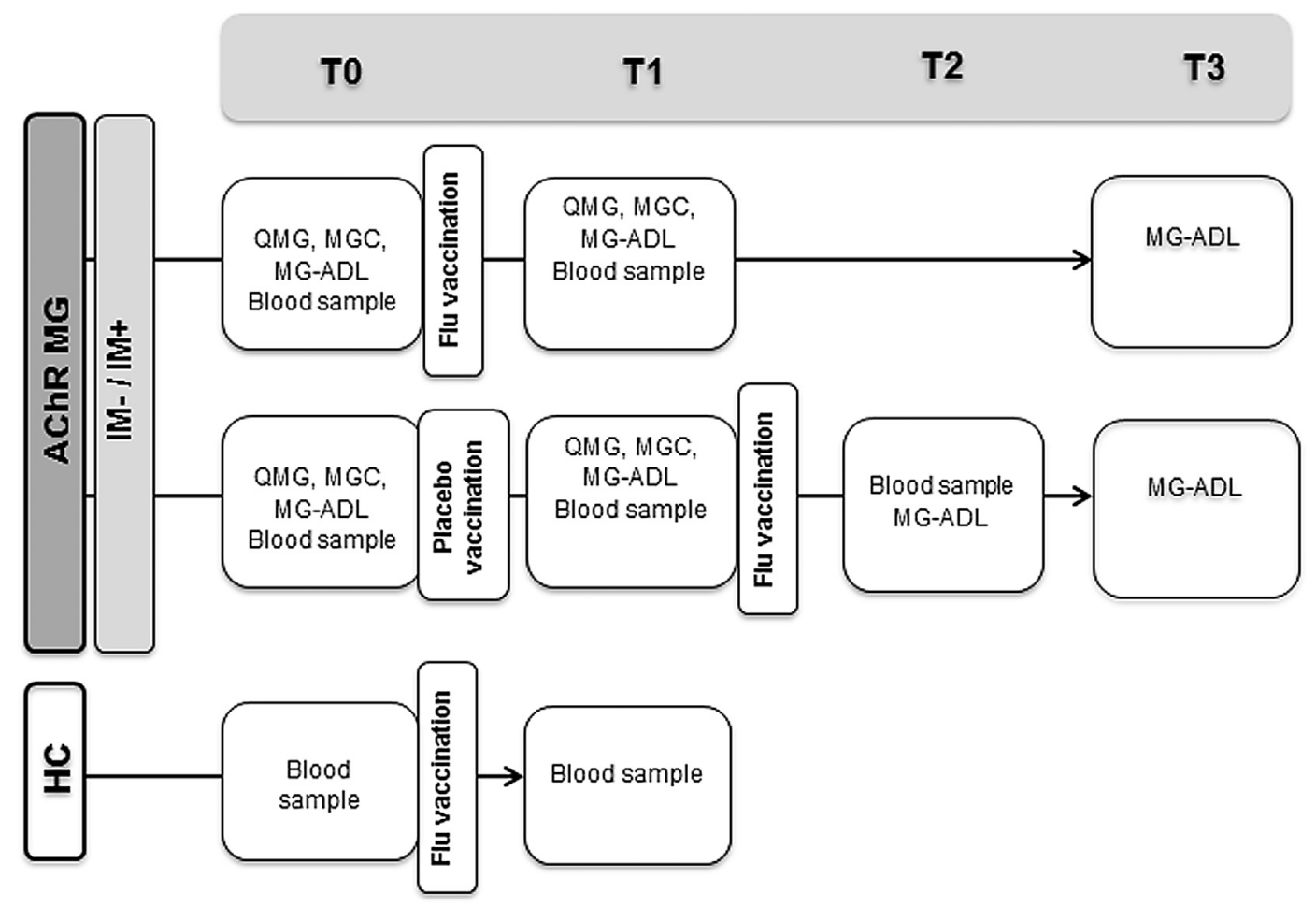

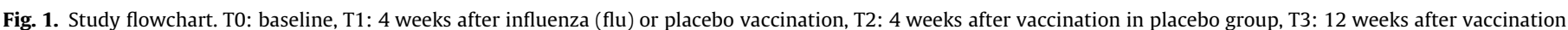

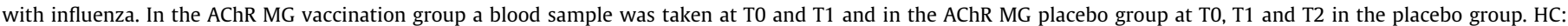
Healthy controls. QMG: Quantitative Myasthenia Gravis score, MGC: Myasthenia Gravis Composite score, MG-ADL: myasthenia gravis activities of daily living score.

of their MG symptoms. Healthy controls were asked for side effects at T1. Fig. 1 shows an overview of the study design.

\subsection{Influenza vaccine}

We used the commercially available influenza vaccine manufactured by Sanofi Pasteur (Vaxigrip, RVG 22306) for the season 2016/2017. One dose of $0.5 \mathrm{~mL}$ contains $15 \mu \mathrm{g}$ haemagglutinin of each of the influenza virus strains in the split inactivated influenza vaccine: A/California/7/2009 (H1N1)pdm09, A/Hong Kong/4801/14 (H3N2) and B/Brisbane/060/08 (B/Victoria/2/87- line). The vaccine was administered intramuscularly, as a bolus, in the non-dominant upper arm.

\subsection{Influenza antibody response}

The primary endpoint of this study was change in titer of antibodies to the flu vaccine strains. A secondary endpoint was the effect of IM on the humoral response. Antibodies to the vaccine strains A/California/7/2009 (H1N1)pdm09, A/Hong Kong/4801/14 (H3N2) and B/Brisbane/060/08 were measured using the hemagglutination-inhibition (HI) assay, according to standard methods at the national influenza center at the Erasmus Medical Center [13]. Titers below the detection limit (i.e. , $\leq 1: 10$ ) were assigned a value of 1:5. Geometric mean titers (GMTs) and seroprotection rates (defined as HI titers $\geq 1: 40$ ) were chosen as the main outcome measures. Seroconversion was defined as a postvaccination $\mathrm{HI}$ titer of at least 1:40 combined with at least a four-fold increase in titer. A non-responder was defined as a post vaccination $\mathrm{HI}$-titer of $<1: 40$.

\subsection{Sampling protocol and clinical scoring}

Another secondary endpoint was a clinical relevant change in clinical scores. We used the QMG, MG Composite (MGC) and the
MG-ADL scores as clinical outcome measures. The QMG is a 13-item scale that measures muscle strength and endurance, ranging from 0 to 39. The MGC is a composite scale selected from existing MG-specific scales (MG-ADL, QMG and Manual Muscle Test (MMT)), ranging from 0 to 50 . The MG-ADL is a scale to assess MG symptoms that patients experience in their daily activities, ranging from 0 to 24 . A change of 2.3 points for the QMG, 3 for the MGC and 2 points for the MG-ADL was considered clinically relevant [14-16]. For all three outcome measures, higher scores indicate a higher clinical severity of MG [14-18].

\subsection{Antibodies against $A C h R$}

The last secondary endpoint was a change in antibodies against AChR. AChR antibody titers were measured with a commercially available radio immunoprecipitation assay (RIA)(RSR Ltd., Cardiff, UK) [19]. Absolute titers were measured using multiple dilutions of each serum sample.

\subsection{Statistical analysis and power}

The study was powered for an expected response rate (i.e. seroprotection rates) of $75 \%$ with a $95 \%$-confidence interval of $63-87 \%$ in MG patients. Herefore, 50 patients with MG were needed. Statistical analysis was performed with Graph-Pad Prism software version 7 and SPSS version 23. In all tests $p<0.05$ was considered statistically significant. Influenza titers were log transformed in order to normalize the data. Comparison for normally distributed numerical variables was done with paired or unpaired T-tests or a one-way analysis of variance (ANOVA).

Influenza virus specific antibody responses were compared between AChR MG patients (with and without immunosuppression) and healthy controls. Within the AChR MG group, patients with and those without thymectomy (Tx) were compared. The AChR antibody titers before vaccination of all AChR MG patients 
were compared to titers 4 weeks after influenza vaccination. The clinical outcome measures were compared between the AChR MG vaccination and placebo group.

\section{Results}

\subsection{Patient characteristics}

Forty-seven patients (53.2\% female, median age 62 years, range $22-74$ years) and 47 healthy controls (76.6\% female, median age 54 years, range 24-66 years) were vaccinated with the seasonal influenza vaccine from October to December 2016. Healthy controls were significantly younger $(p=0.001)$ and were more frequently female $(p=0.02)$ than the MG group. In the MG group, 23 patients randomly received a placebo injection followed by flu vaccination 4 weeks later. Baseline characteristics did not differ between the two MG patients groups that either received first the flu vaccination or the placebo vaccination. The MG group consisted of 29 patients with (IM+) and 18 without (IM-)-) immunosuppressive medication. The IM+ group was significantly older $(\mathrm{p}<0.01)$ than the IM - group and contained more female patients $(p=0.04)$. Disease duration and whether a patient underwent a thymectomy in the past was not significantly different between $\mathrm{IM}-$ and $\mathrm{IM}+$ groups $(\mathrm{p}=0.4$ and $\mathrm{p}=0.16$, respectively). Baseline characteristics are given in Table 1 .

\subsection{Serological response to influenza vaccination}

Upon vaccination the MG group $(\mathrm{n}=47)$ developed a geomean titer (GMT) for all three vaccine strains that was similar to the $\mathrm{HC}$ group (H3N2, $\mathrm{p}=0.2 ; \mathrm{H} 1 \mathrm{~N} 1, \mathrm{p}=0.7$; and $\mathrm{B}$-strain, $\mathrm{p}=0.9$ ) (Fig. 2). The post-vaccination seroprotection and seroconversion rates were comparable between the MG group and HC group for all strains. In the MG group, $40.4 \%$ of all patients (19/47) reached a seroprotective titer for all three strains. In the HC group this was $51 \%(24 / 47)$ (Table 2).

\subsubsection{Influence of use of immunosuppressive medication and thymectomy}

No significant effect on the serological response to influenza vaccination was observed between the IM- $(\mathrm{n}=18)$ and IM+ group $(\mathrm{n}=29)(\mathrm{H} 3 \mathrm{~N} 2, \mathrm{p}=0.2$; H1N1, $\mathrm{p}=0.1$; and B-strain, $\mathrm{p}=0.9)$. The

\section{H3N2 strain}
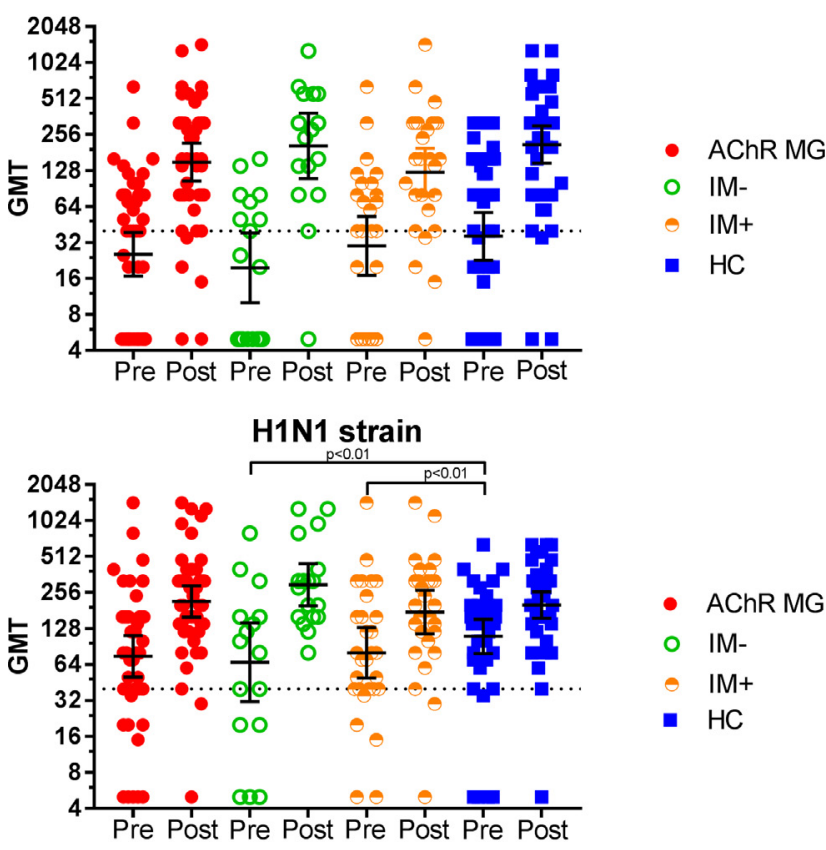

B-strain

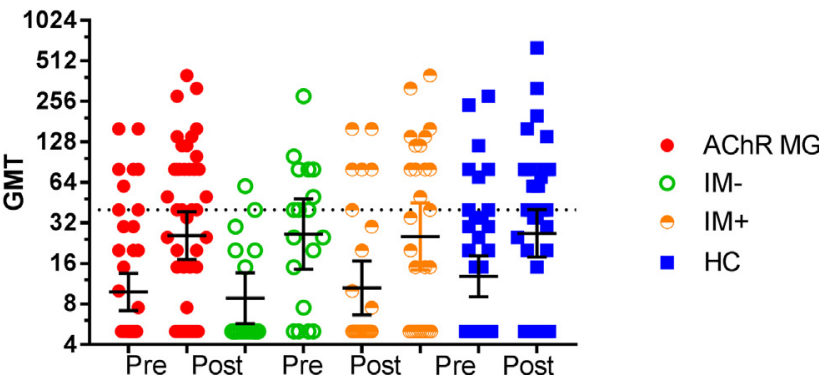

Fig. 2. Response to influenza vaccination. Geomean titers (GMT) of H3N2, H1N1 and B-strain, pre and 4 weeks post-vaccination with a $95 \% \mathrm{CI}$. Groups consist of: 47 AChR MG patients, 18 AChR MG patients without immunosuppressive medication (IM-), 29 AChR MG patients with IM+ and 47 healthy controls. The dotted line is the minimal GMT that is considered as protective (HI-titer 1:40).

Table 2

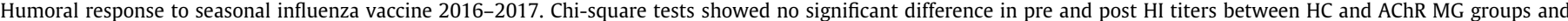
between $\mathrm{HC}$ and IM-/IM+ groups.

\begin{tabular}{|c|c|c|c|c|}
\hline & AChR MG $(n=47)$ & $\mathrm{IM}-(\mathrm{n}=18)$ & $\mathrm{IM}+(\mathrm{n}=29)$ & $\mathrm{HC}(\mathrm{n}=47)$ \\
\hline \multicolumn{5}{|l|}{ H3N2 strain } \\
\hline Pre HI titer $\geq 1: 40-\mathrm{n}(\%)$ & $25(53.2)$ & $8(44.4)$ & $17(58.6)$ & $26(55.3)$ \\
\hline Post HI titer $\geq 1: 40-\mathrm{n}(\%)$ & $42(89.4)$ & $17(94.4)$ & $25(86.2)$ & $44(93.6)$ \\
\hline Pre GMT - value (95\% CI) & $26(17-39)$ & $20(10-39)$ & $30(17-53)$ & $36(23-57)$ \\
\hline Post GMT - value (95\% CI) & $150(104-216)$ & $205(109-384)$ & $124(78-196)$ & $210(147-301)$ \\
\hline Seroconversion - n (\%) & $22(46.8)$ & $11(61.1)$ & $11(37.9)$ & $26(55.3)$ \\
\hline \multicolumn{5}{|l|}{ H1N1 strain } \\
\hline Pre HI titer $\geq 1: 40-\mathrm{n}(\%)$ & $37(78.7)$ & $13(72.2)$ & $24(82.7)$ & $42(89.4)$ \\
\hline Post HI titer $\geq 1: 40-\mathrm{n}(\%)$ & $45(95.7)$ & $18\left(\begin{array}{lll}1 & 0 & 0\end{array}\right)$ & $27(93.1)$ & $46(97.9)$ \\
\hline Pre GMT - value (95\% CI) & $75(50-112)$ & $67(31-143)$ & $80(49-131)$ & $110(79-153)$ \\
\hline Post GMT - value (95\% CI) & $215(159-291)$ & $297(198-446)$ & $176(115-268)$ & $201(156-259)$ \\
\hline Seroconversion - n (\%) & $15(31.9)$ & $6(33.3)$ & $9(31)$ & $9(19.1)$ \\
\hline \multicolumn{5}{|l|}{$B$ - strain } \\
\hline Pre HI titer $\geq 1: 40-\mathrm{n}(\%)$ & $8(17)$ & $2(11.1)$ & $7(24.1)$ & $10(21.3)$ \\
\hline Post HI titer $\geq 1: 40-\mathrm{n}(\%)$ & $22(46.8)$ & $9(50)$ & $13(44.8)$ & $24(51)$ \\
\hline Pre GMT - value (95\% CI) & $10(7-14)$ & $9(6-14)$ & $11(7-17)$ & $13(9-18)$ \\
\hline Post GMT - value (95\% CI) & $26(17-39)$ & $26(15-48)$ & $25(14-45)$ & $27(18-40)$ \\
\hline Seroconversion - $\mathrm{n}(\%)$ & $12(25.5)$ & $7(38.9)$ & $5(17.2)$ & $13(27.7)$ \\
\hline
\end{tabular}


pre-vaccination H1N1 GMT was significantly lower in both the IM - and IM+ groups ( $p<0.01$ for both), but there was no significant difference in post-vaccination GMT compared to the HC group. Seroconversion and post-vaccination seroprotection rates were also similar between $\mathrm{HC}$ and the IM- and IM+ groups (Table 2).

Since the antibody response to influenza is T-cell dependent and a large portion of our patients (42.6\%) underwent a thymectomy in the past (Table 1 ), we tested whether a thymectomy impacted the antibody response. We found no significant difference in pre- $(\mathrm{H} 3 \mathrm{~N} 2, \mathrm{p}=0.7 ; \mathrm{H} 1 \mathrm{~N} 1, \mathrm{p}=0.6 ; \mathrm{B}$-strain, $\mathrm{p}=0.5)$ and post-vaccination GMT (H3N2, p =0.2; H1N1, p =0.4; B-strain, $\mathrm{p}=0.5$ ), neither between patients with and without thymectomy, nor between patients and healthy controls (data not shown).

Both IM use and thymectomy can influence the absolute cell counts of T- and B-cells, therefore, we performed an immunophenotyping in all patients pre- and post-vaccination. Patients of the $\mathrm{IM}+$ group had significantly lower absolute cell counts of CD19+ B-lymphocytes (mean $73 \times 10^{6} / \mathrm{L}, \mathrm{p}<0.001$ ), CD4+ T-lymphocytes (mean $621 \times 10^{6} / \mathrm{L}, \quad \mathrm{p}=0.02$ ), CD8+ T-lymphocytes (mean $245 \times 10^{6} / \mathrm{L}, \mathrm{p}=0.04$ ) and NK-cells (mean $\left.97 \times 10^{6} / \mathrm{L}, \mathrm{p}<0.001\right)$ than patients of the IM- group. However, these values are in the range of healthy controls, except for the CD8+ T-lymphocytes (normal values $260-990 \times 10^{6} / \mathrm{L}$ ). There was no difference in absolute cell counts between the groups with and without thymectomy.

\subsection{Non-responders}

There were 5 non-responders in the MG group to H3N2 vaccination vs. 3 in the HC group, 2 to H1N1 vs. 1 in the HC group, 25 to the B-strain vs. 23 in the HC group. In the IM- group and IM+ group there were 1 and 4 non-responders respectively to H3N2, 0 and 2 respectively to $\mathrm{H} 1 \mathrm{~N} 1,9$ and 16 respectively to the B- strain. The largest difference in response between IM- and IM+ groups was found for the B-strain: 9 non-responders in the IM- group and 16 in the IM+ group, although this apparent difference did not reach statistical significance: $p=0.73$. Of the 16 nonresponders to the B-strain in the IM+ group, 12 used prednisone, 14 used azathioprine and 2 used three types of immunosuppressive medication (prednisone, azathioprine and cyclosporine). Only $1 \mathrm{MG}$ patient and $1 \mathrm{HC}$ were non-responders for all three strains.

\subsection{Clinical scores}

Fig. 4 shows individual clinical scores and changes of the MG vaccination group $(n=24)$ and MG placebo group $(n=23)$ from T0 to T1. Use of IM was comparable (Table 1). Total scores of the three outcome measures were the same before and after vaccination between both groups. In addition, there was no significant change in the mean score or delta of all three outcome measures between $\mathrm{T} 0$ and $\mathrm{T} 1$. The MG-ADL also showed no significant difference 12 weeks (T3) after vaccination in the MG vaccination group compared to $\mathrm{T} 0$ and $\mathrm{T} 1(\mathrm{p}=0.12)$. In the placebo group there was no significant difference between any of the 4 time points at which the MG-ADL was performed (T0-T3) (data not shown).

\subsection{Antibodies against AChR}

No change in antibody titer was observed 4 weeks after influenza vaccination (Fig. 3).

\subsection{Side effects}

The MG vaccination group reported side effects in $30.4 \%(7 / 23)$ at $\mathrm{T} 1$, the placebo group in $37.5 \%(9 / 24)$ at $\mathrm{T} 1(\mathrm{p}=0.6)$. At $\mathrm{T} 2$, 4 weeks after unblinded influenza vaccination of the placebo group

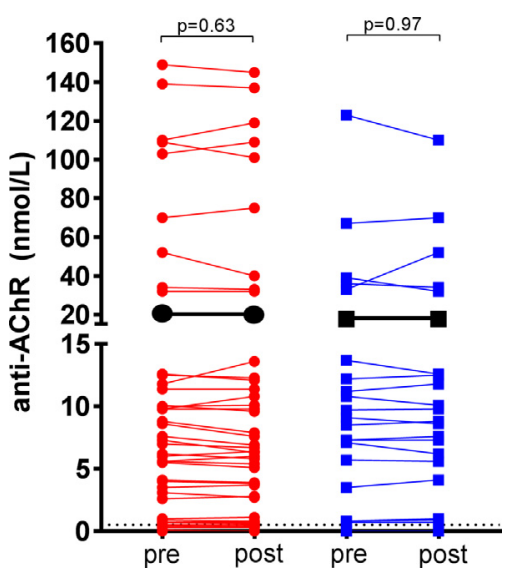

Fig. 3. Anti-AChR antibody concentrations before and 4 weeks after vaccination with influenza in all MG patients and before and 4 weeks after placebo administration. The dotted line indicate the minimal titer that is considered as positive $(0.5 \mathrm{nmol} / \mathrm{L})$. Black: mean titer of the group, individual titers are depicted in colour ( Vaccination group $(n=47)$; $\square$ Placebo group $(n=23)$ ).

$52 \%(12 / 23)$ reported side effects. At T1 healthy controls reported significantly more side effects (70\%; 33/42) than the MG vaccination or placebo group $(\mathrm{p}<0.01)$. The most commonly reported side effects for MG or HC were local redness and soreness at the injection site. No change in MG symptoms was reported in the MG group at T1. In the placebo group, 3 patients reported a mild exacerbation of their MG symptoms during the T1-T2 period. Exacerbation of symptoms lasted 1 day to 1 week after vaccination and did not lead to a change in medication.

\section{Discussion}

In this prospective, double blind, randomized, placebocontrolled study we show that in AChR MG patients influenza vaccination is safe and induces an immune response comparable to that of healthy controls. The study population consisted of patients with stable disease and a stable medication regime in the past 3 months. A seroprotective titer for all three strains of the seasonal influenza vaccine was reached in $40.4 \%$ (19/47) of the AChR MG group and in $51 \%(24 / 47)$ of the HC group. IM or thymectomy status did not significantly influence post vaccination GMT titers. No clinical or immunological exacerbation was found as clinical outcome scores and AChR antibody titers showed no significant changes.

It is generally assumed that patients with an autoimmune disease are more prone to infections, resulting in increased morbidity and mortality [2]. In autoimmune inflammatory rheumatic disease, influenza-vaccinated patients have a lower incidence of pneumonitis, acute bronchitis and viral infections than unvaccinated patients [3]. To our knowledge no such studies have been performed in patients with MG. Recently a randomized controlled trial on influenza vaccination showed that influenza vaccination is safe, based on QMG scores and AChR antibody titers, but without including an healthy control group [8]. Studies on the efficacy of influenza vaccination in rheumatic disease also found that achievement of seroprotection (post HI-titer $\geq 1: 40$ ) is similar to healthy controls, irrespective of medication [3]. In patients with SLE, the response to influenza vaccination is comparable to that of healthy controls [3]. Two studies showed a trend towards a lower response to vaccination in patients who used azathioprine $[20,21]$, which is also commonly used in MG next to corticosteroids. In this study we did not find a significant effect of IM on the humoral response. Due to small size of treatment subgroups and because of frequent 

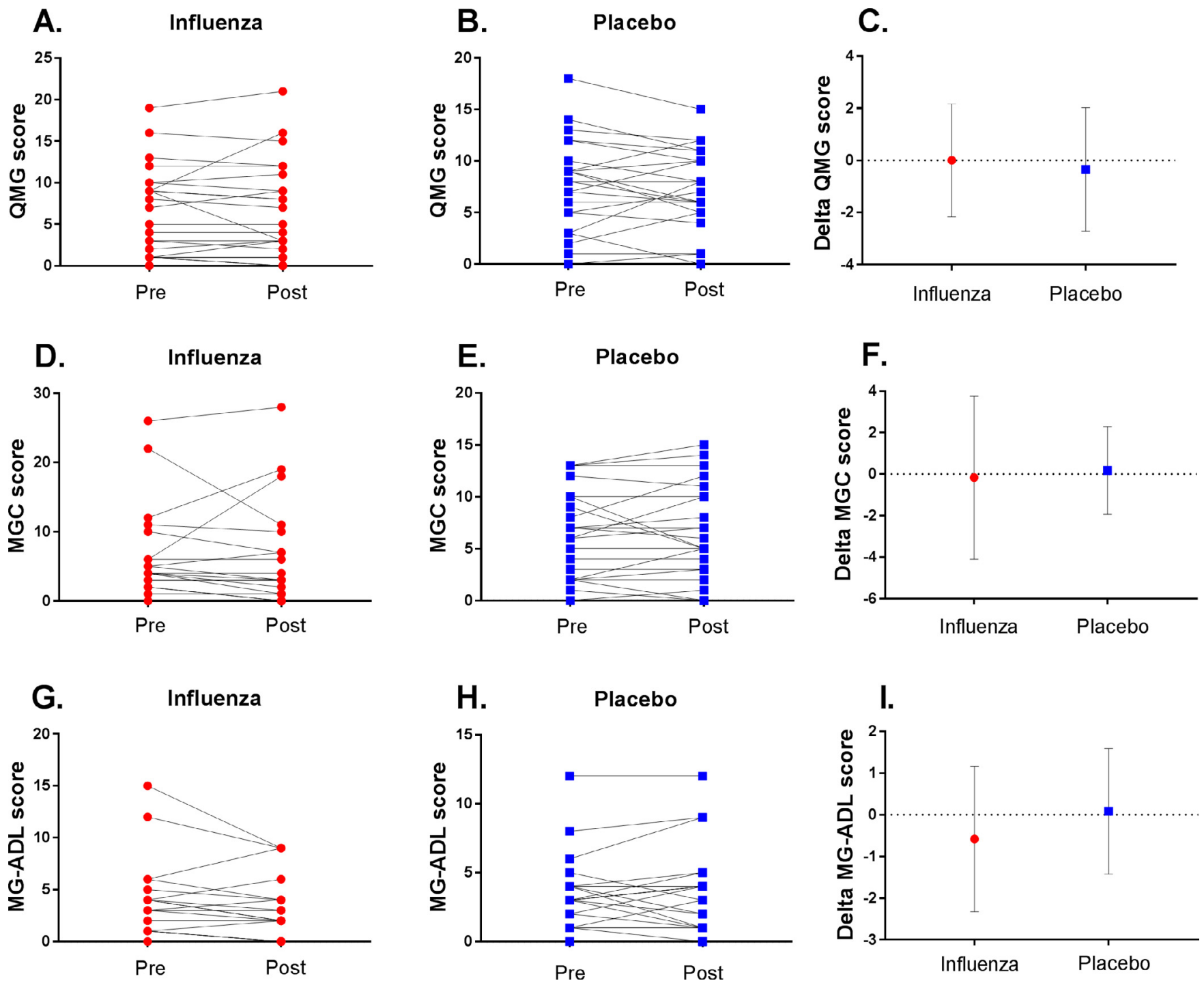

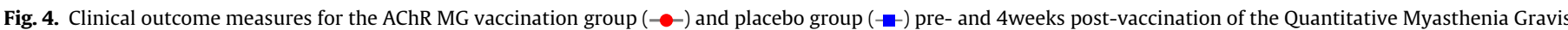

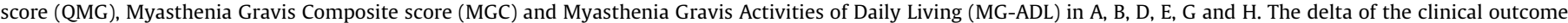
scores are shown in C, F and I. No significant differences were found.

combinations of IM, we could not investigate specific effects of a single drug. In a study on the efficacy and safety of a tetanus vaccination in MG, we found that IM lowers pre- and post-vaccination GMTs, but did not affect the efficacy of the response [9]. This difference might be explained by the type of vaccine that is investigated and the vaccination history of the patients.

Some MG patients chose not to participate out of concern for an exacerbation of their symptoms. Even in our trial participants, only two-thirds had obtained an influenza vaccination in previous years, similar to the frequency of our healthy controls.

The tetanus revaccination study in AChR MG patients showed a small but statistically significant increase of the QMG score of 1 point at 4 weeks, which is far less than the 2.3 points that is generally accepted as the minimal clinically relevant difference. A recent study indicated that an exacerbation of MG is more likely after an influenza-like infection or a common cold, than following an influenza vaccination (10/25 (40\%) and 15/96 (15.6\%) vs. $2 / 133$ (1.5\%)) [7]. In line with our results, no clinical exacerbation was found in patients with RA and SLE following influenza vaccination [3].

Interestingly, unblinded influenza vaccination of MG patients in $\mathrm{T} 1-\mathrm{T} 2$ resulted in more reported side effects and a higher incidence of self-reported aggravation of MG symptoms than blinded vaccination or placebo injection. This may be explained by the presence of a prejudice among MG patients that vaccination might be harmful, leading to increased reporting of subjective complaints.

\subsection{Strengths and limitations}

The main strengths of this study are its placebo-controlled, double blind, randomized design and the systematic assessment of multiple relevant measures of clinical disease severity at multiple time points up to twelve weeks.

Limitations are the exclusion of patients with severe or unstable MG and patients using high doses of corticosteroids. Therefore we cannot draw a conclusion on the safety and efficacy of vaccination in these groups. Although the study was not powered to detect small changes in clinical outcomes, none of these measures show a trend indicating a possible negative effect.

Theoretically, the unblinded nurses may have caused unblinding of patients, but they specifically ensured that patient blinding was maintained during injection. Furthermore, clinical outcome measures, which are likely the most susceptible to unblinding were taken before unblinding the patients 4 weeks after the 
injection. Median age of healthy controls was lower, which might result in an stronger humoral response. However, no significant post-vaccination differences were observed between MG and HC groups.

\section{Conclusion}

The antibody response to an influenza vaccination in patients with mild to moderate MG is similar as in healthy subjects, and not affected by the use of immunosuppressive medication. Influenza vaccination did not induce any immunological or clinical exacerbation of MG.

\section{Acknowledgements}

We would like to thank Martha Mosselman and Marjolein Heur-Neuman, research nurses, for their efforts in coordinating the study.

\section{Author contributions}

1) conception and design of the study: ES, FK, GR, JV.

2) acquisition and analysis of data: ES, IA, RM, RB, JB, MT, JB, JV.

3) drafting a significant portion of the manuscript or figures: ES, MT, FK, GR, JV.

4) Approval of finale version: all authors have approved the final version of the article.

\section{Conflicts of interest}

J.J.G.M.V. received grants from Prinses Beatrix Spierfonds, NIH, and a FP7 European grant (\#602420), outside the submitted work. J.J.G.M.V. has participated in research collaboration and consultancy with Argenx, Alexion and Rapharma. The Leiden University Medical Center (LUMC) received royalties from IBL for antibody tests, which were not used in this study. All reimbursements were received by the LUMC.

\section{References}

[1] Gilhus NE, Verschuuren JJ. Myasthenia gravis: subgroup classification and therapeutic strategies. Lancet Neurol 2015;14:1023-36.

[2] Meyer-Olson D, Witte T. Immunology: prevention of infections in patients with autoimmune diseases. Nat Rev Rheumatol 2011;7:198-200.
[3] Westra J, Rondaan C, van Assen S, Bijl M. Vaccination of patients with autoimmune inflammatory rheumatic diseases. Nat Rev Rheumatol 2015;11:135-45.

[4] Gilhus NE, Romi F, Hong Y, Skeie GO. Myasthenia gravis and infectious disease. J Neurol 2018.

[5] Auriel E, Regev K, Dori A, Karni A. Safety of influenza and H1N1 vaccinations in patients with myasthenia gravis, and patient compliance. Muscle Nerve 2011;43:893-4.

[6] Zinman L, Thoma J, Kwong JC, Kopp A, Stukel TA, Juurlink DN. Safety of influenza vaccination in patients with myasthenia gravis: a population-based study. Muscle Nerve 2009;40:947-51.

[7] Seok HY, Shin HY, Kim JK, Kim BJ, Oh J, Suh BC, et al. The impacts of influenza infection and vaccination on exacerbation of myasthenia gravis. J Clin Neurol 2017;13:325-30.

[8] Tackenberg B, Schneider M, Blaes F, Eienbroker C, Schade-Brittinger C, Wellek A, et al. Acetylcholine receptor antibody titers and clinical course after influenza vaccination in patients with myasthenia gravis: a double-blind randomized controlled trial (ProPATIent-Trial). EBioMedicine 2018.

[9] Strijbos E, Huijbers MG, van Es IE, Alleman I, van Ostaijen-Ten Dam MM Bakker J, et al. A prospective, placebo controlled study on the humoral immune response to and safety of tetanus revaccination in myasthenia gravis. Vaccine 2017;35:6290-6.

[10] Klinkenberg RE, Gelinck LB. Influenza vaccination in immunocompromised patients. Ned Tijdschr Geneeskd 2014;158:A7574.

[11] Kassianos G, Blank P, Falup-Pecurariu O, Kuchar E, Kyncl J, De Lejarazu RO, et al. Influenza vaccination: key facts for general practitioners in Europe-a synthesis by European experts based on national guidelines and best practices in the United Kingdom and the Netherlands. Drugs in Context 2016;5:212293.

[12] Carr AS, Cardwell CR, McCarron PO, McConville J. A systematic review of population based epidemiological studies in Myasthenia Gravis. BMC Neurol 2010;10:46.

[13] Vogtlander NP, Brown A, Valentijn RM, Rimmelzwaan GF, Osterhaus AD. Impaired response rates, but satisfying protection rates to influenza vaccination in dialysis patients. Vaccine 2004;22:2199-201.

[14] Muppidi S. The myasthenia gravis-specific activities of daily living profile. Ann N Y Acad Sci 2012;1274:114-9.

[15] Bedlack RS, Simel DL, Bosworth H, Samsa G, Tucker-Lipscomb B, Sanders DB. Quantitative myasthenia gravis score: assessment of responsiveness and longitudinal validity. Neurology 2005;64:1968-70.

[16] Burns TM, Conaway M, Sanders DB. The MG Composite: a valid and reliable outcome measure for myasthenia gravis. Neurology 2010;74:1434-40.

[17] Barohn RJ, McIntire D, Herbelin L, Wolfe GI, Nations S, Bryan WW. Reliability testing of the quantitative myasthenia gravis score. Ann N Y Acad Sci 1998:841:769-72.

[18] Burns TM, Conaway MR, Cutter GR, Sanders DB. Construction of an efficient evaluative instrument for myasthenia gravis: the MG composite. Muscle Nerve 2008;38:1553-62.

[19] Vincent A, Newsom-Davis J. Acetylcholine receptor antibody as a diagnostic test for myasthenia gravis: results in 153 validated cases and 2967 diagnostic assays. J Neurol Neurosurg Psychiatry 1985;48:1246-52.

[20] Holvast A, Huckriede A, Wilschut J, Horst G, De Vries JJ, Benne CA, et al. Safety and efficacy of influenza vaccination in systemic lupus erythematosus patients with quiescent disease. Ann Rheum Dis 2006;65:913-8.

[21] Abu-Shakra M, Press J, Varsano N, Levy V, Mendelson E, Sukenik S, et al. Specific antibody response after influenza immunization in systemic lupus erythematosus. J Rheumatol 2002;29:2555-7. 